\title{
A Prototype System for Autism Rehabilitation Based on Broken Mirror Theory
}

\author{
ZHU Huaping ${ }^{1, a}$, ZHAO Ming ${ }^{1, b}$ and SUN Yaoru ${ }^{1, c}$ \\ ${ }^{1}$ Siping Road 1239, Tongji University, Shanghai City, China \\ ahuapingzhu@tongji.edu.cn, bzhaom@tongji.edu.cn, cyaoru@tongji.edu.cn
}

\begin{abstract}
Keywords: Mirror neuron system, Brain-computer interface, mu suppression, Computer-based intervention, Electroencephalograph.

Abstract. It is seems that the dysfunction of the human mirror neuron system plays an important role in the autism spectrum disorder (ASD). In this paper, we described a novel information system for autism therapy by means of mirror neural training induced by virtual reality in brain-computer interfaces. This system was designed to improve the mirror neuron system's functions of autistic individuals as well as the ability to understand other's intentions by self-interaction in a virtual reality training environment, in which some trials for training such as action observation, facial expression recognition and action imitation were designed.
\end{abstract}

\section{Introduction}

Children with autism spectrum disorder (ASD) have developmental disability that is characterized by social interaction deficits, impaired communication skills, pragmatic language, and stereotyped patterns of behavior. As an incurred disease, the current treatments for individuals with ASD mainly includes behavioral interventions, such as psychology, genetics and neuroscience approach. The major cause of ASD is still unknown, but a large number of studies have suggested that individuals with ASD have a deficit in the human mirror neuron system (hMNS), the so-called "the broken mirror theory" might account for their communication difficulties[1-4]. Accordingly, some methods as to improve mirror neuron functioning by socialization practices might be a promising approach for the autism therapy.

Information system is common used in healthcare delivery and management for its computing power that can accomplish many tasks autonomously, quickly and efficiently. The behavioral interventions are currently the primary treatments for individuals with ASD, but many computer-based interventions (CBI) can not monitor the neural activity. Because brain-computer interface (BCI) systems can connect the brain and an external device, more and more BCI devices are directed at assisting, augmenting, or repairing human cognitive or sensory-motor functions. It is widely accepted that mu suppression is an index for human mirror neurons activities [5,6], as well as can be measured in a BCI systems. Previous research[7] has proved that EEG mu rhythm and imitation impairments in individuals with autism spectrum disorder. We have provided a new idea that use of computer-based interventions (CBI) to improve human MNS' functions in autistic individuals by using a BCI system with virtual reality technology[8]. On the other hand, the hMNS modulated by motor learning may provide a promising approach for the autism to learn and develop social communications in an information system. Accordingly, we developed some modules, such as EEG data collection, analysis and computing, in our prototype system which described a novel information system for autism therapy by means of mirror neural training induced by virtual reality in brain-computer interfaces.

\section{Action-related information repository for training}

In most of information systems for treating autistic children, the stimulus used to train sociability formed by voices, texts, pictures and videos, which show the content are mostly associated with action, facial expression, social-contact process and combinations of each other. In the action-related information repository of our system, the interventional trial and strategy can be designed for use in 
professional development training in order to promote the rehabilitation of hMNS' function correctly as well as for the redesign and assessment by expert knowledge or online statistical analysis. Some interventional trials and strategies in this prototype show in Fig. 1.

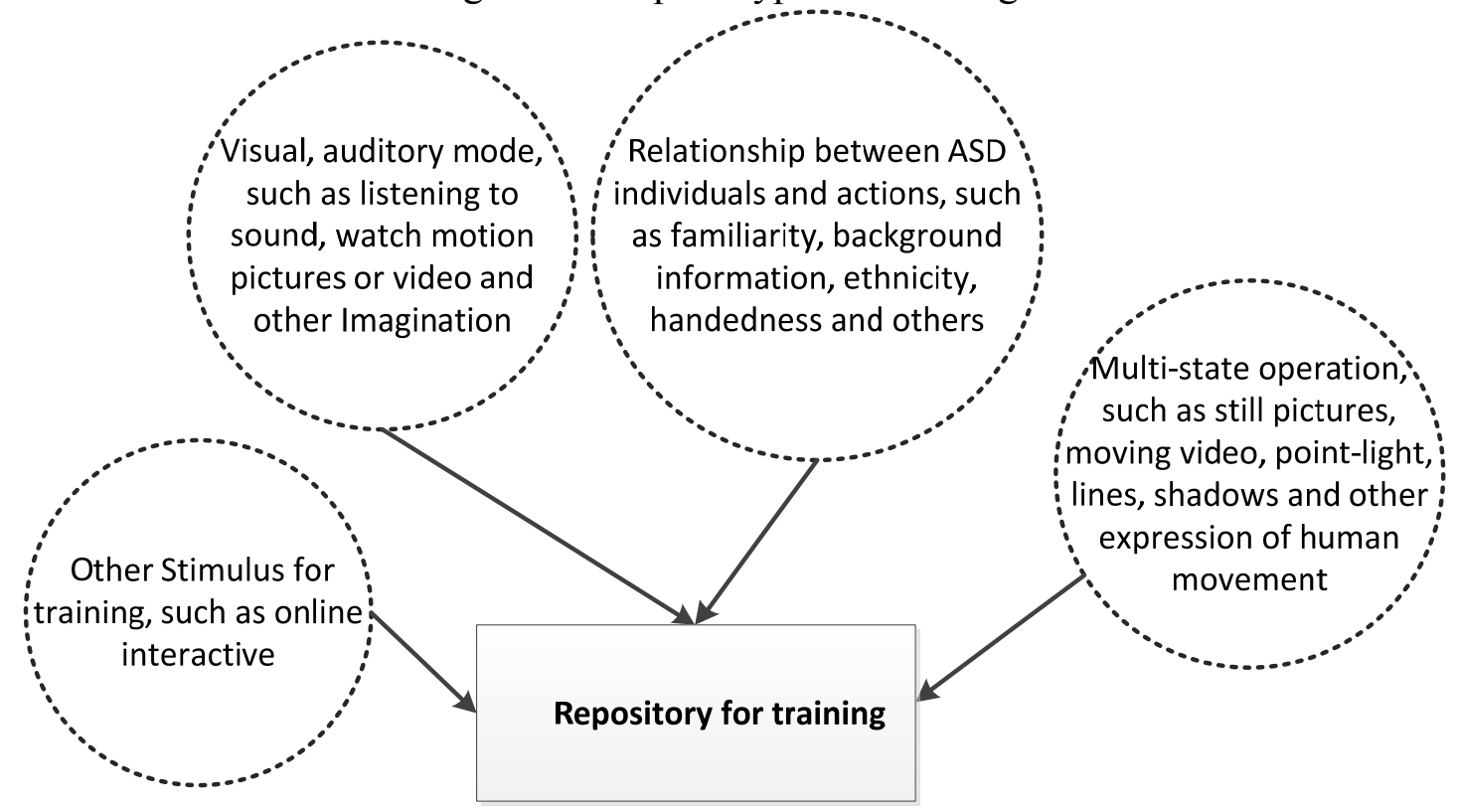

Fig.1 Action-related information repository for training

\section{EEG Calculation}

Four bands EEG data (i.e., $\delta: 1-3 \mathrm{~Hz}, \theta: 4-7 \mathrm{~Hz}, \alpha / \mu: 8-13 \mathrm{~Hz}, \beta: 14-30 \mathrm{~Hz}$ ) were mostly calculated in a large number of BCI systems. As an index for hMNS' activity responding to action-related stimuli observation, the mu suppression can be measured by a BCI system. Accordantly, the spectral power in the four bands (i.e., $\delta, \theta, \alpha / \mu$ and $\beta$ ) and their rations (i.e., $(\theta+\alpha) / \beta, \alpha / \beta,(\theta+\alpha) /(\alpha+\beta)$ and $\theta / \beta)$ were also calculated as features for brain activity as well as interactive visualization environment.

The choice of the baseline is very important for mu suppression computing, some well-designed stimulus (e.g., several point motions that were visually equivalent to the trajectory taken by training stimulus) were also considered in this system. The mu suppression was calculated as the power during each training condition relative to the power during the baseline. Data are presented as ratios to minimize individual variability in absolute mu power that may result from differences in electrode impedance or scalp thickness, as opposed to mirror neuron activity. Because the ratio data are inherently non-normal as a result of lower bounding, a log transform was used for analysis. A log ratio of less than zero indicates suppression whereas a value of zero indicates no suppression and values greater than zero indicate enhancement [9].

\section{System Framework and related modules}

Based on the "broken mirror" theory, this systems can provide an assessment report for autism symptoms by monitor the changes in the ratio between energy and frequency of EEG, especially the mu suppression as hMNS' activity. The overall framework of this system shown in Fig. 2.

It should be noted that little machine system can be evaluated autistic symptoms, therefore expert evaluation method is currently the main means of autism diagnosis. Theoretically, there may be a difference of EEG rhythm between normal and autism children when they observe same action-related stimulus. In our prototype system, EEG rhythm features can be classified by some EEG pattern classification methods (such as Gaussian, SVM, etc.). In order to evaluate indicators as autism symptoms, four frequency $\operatorname{EEG}(\delta, \theta, \alpha / \mu, \beta)$ and their ratios are also calculated and shown in figure 3 in this system. 


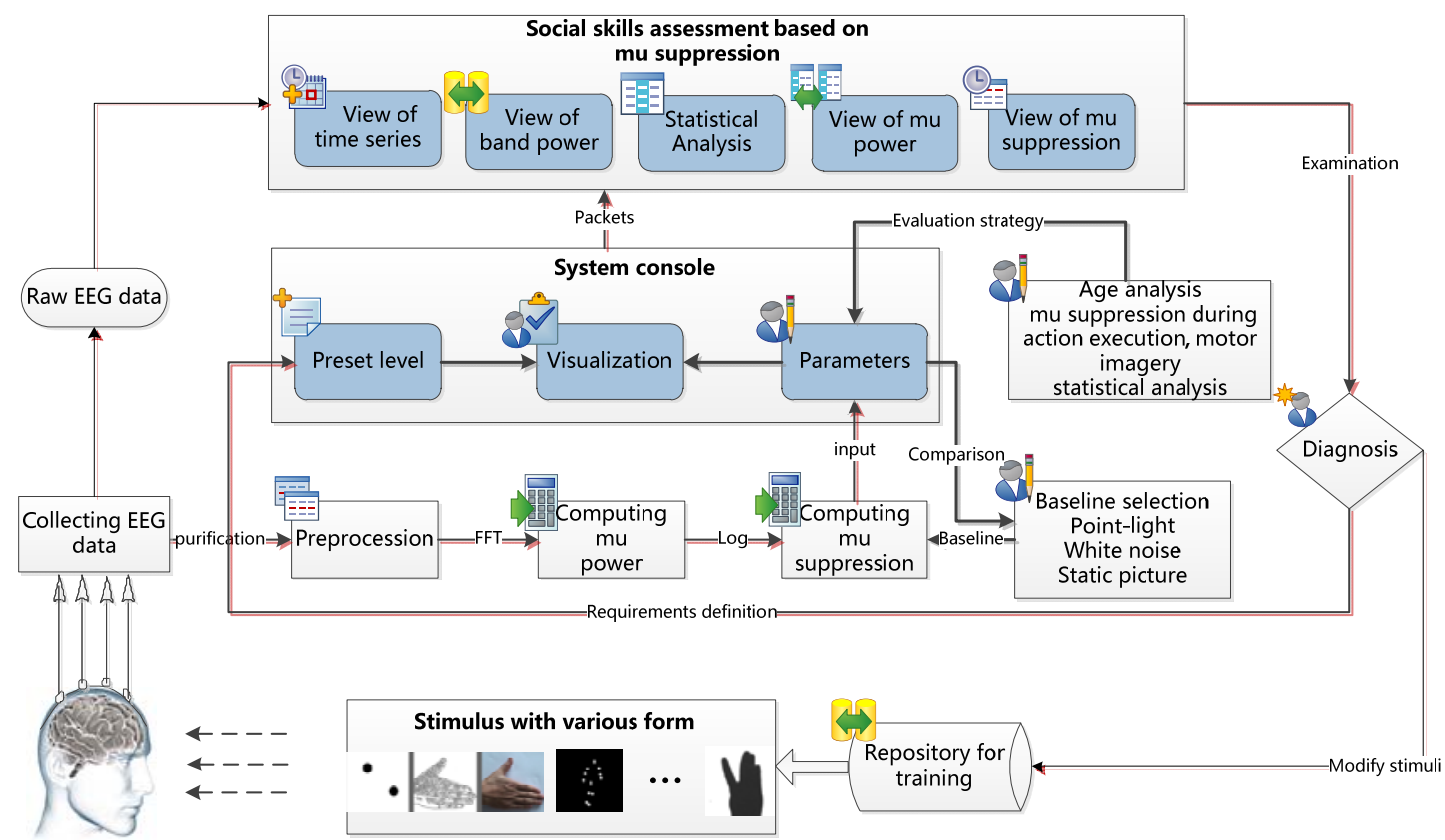

Fig.2 Framework of interventional treatment system for autism rehabilitation

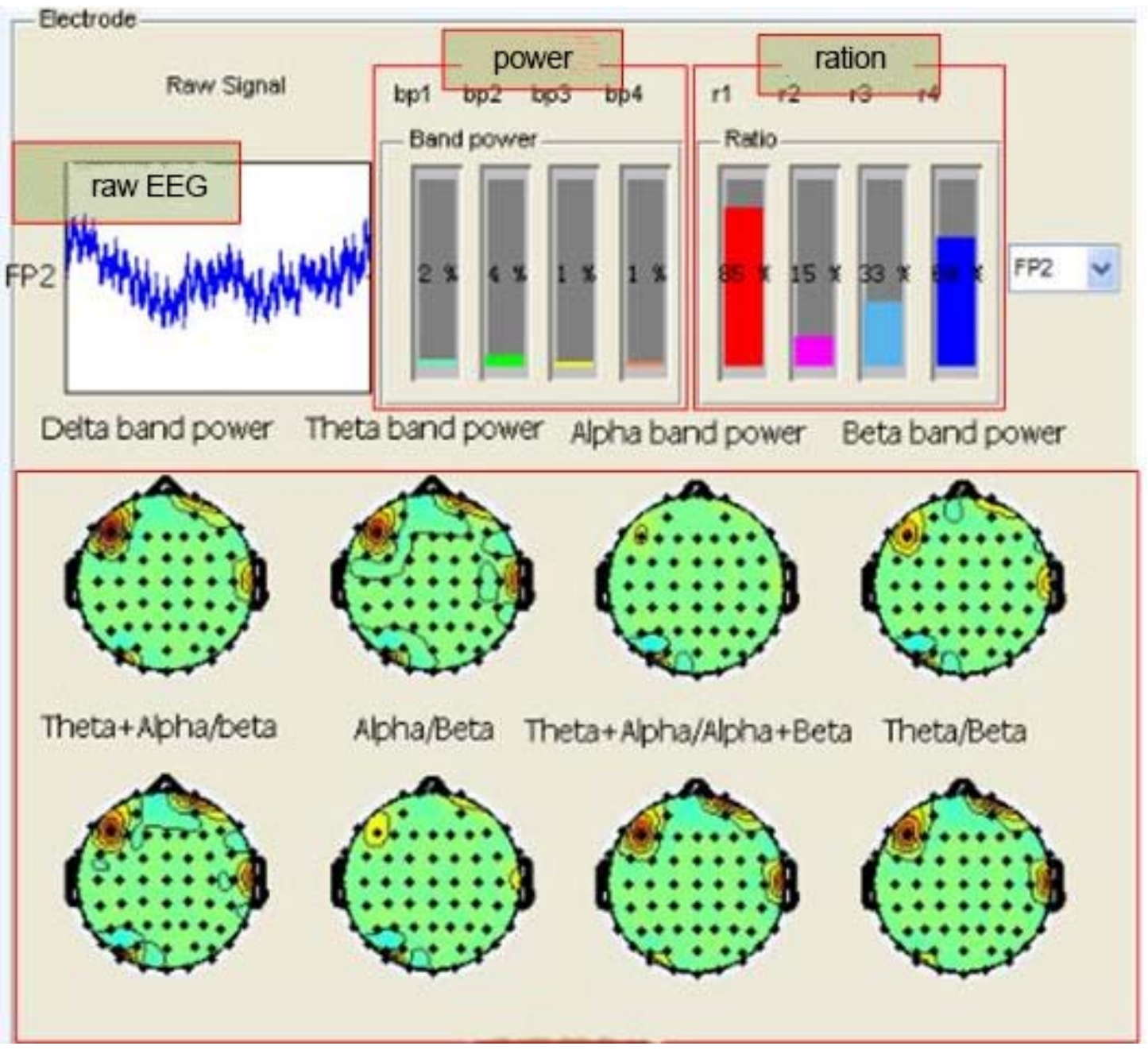

Fig. 3 Display of interventional treatment system for autism rehabilitation. bp1, bp2, bp3 and bp4 represent the four band energy $\delta, \theta, \alpha / \mu$ and $\beta$ rhythm, r1, r2, r3 and $\mathrm{r} 4$ represent the four ratios $(\theta+\alpha) / \beta, \alpha / \beta,(\theta+\alpha) /(\alpha+\beta)$ and $\theta / \beta$.

It should be noted that eight EEG characteristic parameters displayed in Figure 3 were collected from normal subjects in different mental states (such as alertness and fatigue). Whether EEG 
frequency analysis can react the degree of autism symptom is an open question, this prototype system provided a promising approach for the autism therapy.

\section{Conclusion}

Autism pathogenesis, biologically-plausible computing models, and their application systems of computer-based interventions method is one of the cutting-edge research fields in neurophysiology, cognitive science and informatic technology with both important theoretical and applicational values. A large number of studies have shown that the human mirror neuron system pay a core role in action understanding, as well as hMNS' dysfunction named "broken mirror" may account for the symptom with social deficit in autism individuals. Based on the "broken mirror theory", we proposed a novel training program from our interdisciplinary research to improve the hMNS' functions of autistic individuals by using a BCI system with human-machine interaction (HMI) technology. We believe that the system in this work can be help for the future study on autism rehabilitation.

\section{Acknowledgements}

This work was financially supported by the Key Projects in the National Science and Technology Pillar Program during the twelfth Five-year Plan Period (2011BAK02B04), the General Financial Grant from the China Postdoctoral Science Foundation (2013M541541) and Grants from the National Natural Science Foundation of China (51478363 and 61173116)

\section{References}

[1] A.F. Hamilton: Research review: Goals, intentions and mental states: Challenges for theories of autism. Journal of Child Psychology and Psychiatry Vol.50 (2009), p. 881-892.

[2] R.M. Le-Bel, J.A. Pineda and A. Sharma: Motor - auditory - visual integration: The role of the human mirror neuron system in communication and communication disorders. Journal of communication disorders Vol. 42(2009), p. 299-304.

[3] V.S. Ramachandran and J.A. Pineda: EEG evidence for mirror neuron dysfunction in autism spectrum disorders. Cognitive Brain Research Vol.24 (2005), p. 190-198.

[4] A. Hamilton: Reflecting on the mirror neuron system in autism: A systematic review of current theories. Developmental Cognitive Neuroscience Vol.3 (2013), p. 91-105.

[5] L.M. Oberman, J.P. Mccleery, V.S. Ramachandran, et al: EEG evidence for mirror neuron activity during observation of human and robot actions: Toward an analysis of the human qualities of interactive robots. Neurocomputing Vol.70 (2007), p. 2194-2203.

[6] A. Perry, S. Bentin: Mirror activity in the human brain while observing hand movements: A comparison between EEG desynchronization in the $\mu$-range and previous fMRI results. Brain Research Vol.1282 (2009), p.126-132.

[7] R. Bernier, G. Dawson, S. Webb, et al: EEG mu rhythm and imitation impairments in individuals with autism spectrum disorder. Brain and cognition Vol.64 (2007), p. 228-237.

[8] H.P. Zhu, Y.R. Sun, J.H. Zeng, et al: Mirror neural training induced by virtual reality in brain computer interfaces may provide a promising approach for the autism therapy. Medical Hypotheses Vol.76 (2011), p. 646-647.

[9] H.P. Zhu, Y.R. Sun and F. Wang: Electroencephalogram evidence for the activation of human mirror neuron system during the observation of intransitive shadow and line drawing actions. Neural Regeneration Research Vol.8 (2013), p. 251-257. 